\title{
Saúde Ambiental na América Latina e no Caribe: numa encruzilhada
}

\section{Environmental Health in Latin America and the Caribbean: at the Crossroads}

\author{
Mirta Roses Periago \\ Diretora da Organização Pan-Americana da Saúde e Diretora Re- \\ gional para as Américas da Organização Mundial da Saúde, Wa- \\ shington DC \\ Endereço: 525 23rd Street, N. W., Washington, D. C. 20037-2895, \\ E. U. A.

\section{Luiz Augusto Galvão} \\ Gerente da Área de Desenvolvimento Sustentável e Saúde Am- \\ biental, Organização Pan-Americana da Saúde, Washington DC \\ Endereço: 525 23rd Street, N. W., Washington, D. C. 20037-2895, \\ E. U. A. \\ E-mail: heqळpaho.org

\section{Carlos Corvalán} \\ Coordenador, Saúde Ocupacional e Ambiental, Organização Mun- \\ dial da Saúde, Genebra \\ Endereço: 20, Avenue Appia, $\mathrm{CH}-1211$ Geneva 27, Suiça. \\ Jacobo Finkelman \\ Representante da OPAS/OMS. México. \\ Endereço: 525 23rd Street, N. W., Washington, D. C. 20037-2895, \\ E. U. A.
}

\section{Resumo}

É inegável que a discussão sobre saúde, meio ambiente e desenvolvimento sustentável tem progredido muito em décadas recentes. Contudo, ganhos globais não têm sido distribuídos de maneira uniforme, deixando grandes grupos populacionais excluídos, com conseqüências negativas à saúde. Também estamos começando a reconhecer problemas globais emergentes que causam impactos locais significativos, principalmente em populações pobres, tanto em áreas rurais como urbanas. A saúde ambiental está numa encruzilhada, em que novos modelos e parcerias são necessários. Este artigo explora essas questões especificamente em relação aos países latino-americanos e caribenhos. Palavras-chave: Riscos Ambientais; Saúde Ambiental; América Latina; Caribe; Desigualdades. 


\section{Abstract}

There has been undeniable progress in addressing health, environment and sustainable development in recent decades. Yet, global gains have not been distributed equally, leaving major populations groups excluded, with negative consequences to health. We are also beginning to recognize emerging global problems with significant local impacts, mostly in impoverished populations, both in rural and urban settings. Environmental health is at the crossroads, where new models and partnerships are required. This paper explores these issues with specific reference to the Latin American and Caribbean countries.

Keywords: Environmental Risk; Environmental Health; Latin America; Caribbean; Inequalities.

\section{Introdução}

A história recente da saúde ambiental global tem sido marcada por ciclos de grande visibilidade em alternância com outros de obscuridade e descaso. Por um lado, houve incontestáveis progressos em áreas conceituais e metodológicas que tornaram a saúde ambiental um dos pilares do desenvolvimento sustentável e, mais recentemente, um componente essencial dos direitos civis e da segurança humana. No entanto, por outro lado, os ganhos na área de intervenções e aplicações específicas ainda são bastante limitados, expondo grandes grupos populacionais a uma série de riscos ambientais inaceitáveis e injustos, com conseqüências negativas à saúde. Muitos desses problemas, ao invés de serem solucionados, continuam a aumentar. Além disso, hoje nos defrontamos com evidências cada vez maiores das conseqüências que riscos emergentes trazem para a saúde - desde o impacto que ecossistemas em deterioração causam em comunidades locais até as mudanças climáticas, que provocam impactos globais. Tais problemas emergentes também acentuarão o impacto de problemas já existentes, tais como mudanças na disponibilidade de água ou no alcance e na sazonalidade de vetores de doenças.

A lacuna entre conhecimento e ação é especialmente ampla nos países latino-americanos e caribenhos. A região contém áreas cuja biodiversidade está entre as mais ricas do planeta, mas, também, entre as mais ameaçadas de sofrer perdas permanentes. Contém apenas cerca de $14 \%$ da população mundial (United Nations, 2005), mas essa população está densamente concentrada em áreas urbanas. 0 padrão predominante de desenvolvimento continua a transformar ecossistemas de forma irreversível, expandindo suas fronteiras, colonizando novos territórios e gerando projetos agrícolas, industriais, de extração e de energia que são lucrativos em curto prazo, mas exploram os recursos naturais negligentemente. 0 crescente esgotamento dos ecossistemas tem sido parcialmente benéfico para alimentar o crescimento populacional, mas nem sempre tem resultado em melhor alimentação, e o impacto negativo sobre outros ecossistemas tem sido substancial. Estes incluem ecossistemas litorâneos degradados, que ameaçam a conservação da água e a proteção da biodiversidade. Apenas recentemente começamos a compreender os grandes impactos 
que a saúde de nosso meio ambiente ameaçado sofrerá em médio e longo prazo (WHO, 2005). 0 modelo atual de crescimento econômico é injusto e insustentável, fomentando padrões de consumo que têm fortes preferências por bens materiais de vida curta que se tornam obsoletos rapidamente, com grande conteúdo de resíduos não-biodegradáveis. Ao mesmo tempo, a polarização inaceitável na distribuição de renda e de riquezas permanece e até mesmo aumentou, na maioria dos países, correlacionando-se fortemente às grandes disparidades em termos de desfechos em saúde (Leff, 1994).

Nas três últimas décadas, os países conseguiram chegar a um consenso em relação à urgência de se considerar alguns dos desequilíbrios ambientais e outros problemas relacionados como determinantes fortemente associados à deterioração da qualidade de vida e da saúde humana. Isso foi realizado em vários fóruns como, por exemplo, em conferências da ONU sobre Meio Ambiente e Desenvolvimento, e nas conferências mais importantes do setor de saúde, que focalizaram Cuidados Básicos com a Saúde e Promoção de Saúde. Esse movimento forneceu novos modelos à saúde ambiental, como a abordagem de "ambientes saudáveis”, com suas inúmeras aplicações valiosas e criativas: por exemplo, escolas saudáveis, locais de trabalho saudáveis, comunidades saudáveis, mercados e feiras saudáveis e cidades saudáveis.

Entretanto, o cenário global mudou muito nesses últimos 30 anos. A interdependência e a assimetria entre as nações continuam a ser expressões da globalização, com impactos multidimensionais sobre toda a estrutura da sociedade. Uma parte dessa equação é o direito à saúde e a um meio ambiente em equilíbrio. Na década de 1990, a Região das Américas considerou essas questões como elementos críticos em muitas reuniões regionais, fornecendo conteúdo ao conceito emergente de segurança humana (Organization of American States, 2001). Esse conceito transcende as visões tradicionais que o colocam exclusivamente dentro da esfera da ação militar e policial, da luta contra o terrorismo e o crime organizado. Na saúde, essa nova abordagem assume uma visão mais ampla de diretos civis, focalizando determinantes econômicos, sociais e ambientais, visando a uma vida mais justa para to- dos. Esse modelo aborda a segurança não apenas de nações, mas de pessoas, famílias e indivíduos.

Dia após dia, questões que ameaçam a paz e a estabilidade mundiais estão se tornando mais relevantes e centrais para a sociedade global, abarcando problemas como o aumento da violência urbana, do crime organizado, do terrorismo, da fome e de desastres naturais e provocados pelo homem. Também incluídas nessa categoria encontram-se as questões ambientais, como as mudanças climáticas e a degradação de ecossistemas. De fato, as doenças ambientais mais antigas ainda são nossas principais preocupações, quando consideramos as invasões dos seres humanos e as modificações de ecossistemas. Esse é o caso de muitas doenças zoonóticas novas ou re-emergentes, incluindo a possibilidade de que o vírus da gripe aviária passe para os humanos e o estabelecimento da transmissão de pessoa para pessoa. Essas questões demandam que as agendas e as estratégias de ação sejam repensadas em todos os níveis (Annan, 2004).

\section{Um Grande Impacto sobre a Saúde Advindo de um Meio Ambiente em Processo de Degradação}

De acordo com estimativas da OMS, cerca de um quarto da responsabilidade total global pelas doenças pode ser atribuído ao meio ambiente em modificação, embora na América Latina e no Caribe a fração seja aproximadamente um quinto ${ }^{1}$. No início do século XXI, os números relacionados a fornecimento de água potável e cobertura sanitária na América Latina e no Caribe eram $89 \%$ e $75 \%$, respectivamente (WHO/UNICEF, 2005). Quando números absolutos são considerados, a situação torna-se mais surpreendente. Aproximadamente 6o milhões de pessoas não têm acesso a um fornecimento seguro de água potável e 137 milhões não têm instalações para saneamento e eliminação de esgoto e outros resíduos. A Região é claramente marcada por sérias desigualdades no que se refere ao acesso à água - geralmente relacionadas ao nível de renda e local de residência, sendo que a situação mais crítica pode ser encontrada em áreas rurais e periurbanas.

Essas desigualdades não se limitam ao acesso a

1 WHO - WORLD HEALTH ORGANIZATION. Unpublished data. Geneva, 2005. 
serviços de saneamento básico. Estima-se que o ar respirado por cerca de 100 milhões de pessoas, especialmente em áreas urbanas, não atinja os padrões mínimos de qualidade e segurança (PAHO/WHO, 2004). Ademais, muitos trabalhadores e populações de baixa renda são envenenados pela exposição a produtos químicos perigosos, devido à inexistência de medidas eficazes de proteção e controle. A poluição do ar em recintos fechados, proveniente do uso de biomassa e outros combustíveis sólidos, é particularmente perigosa à saúde das crianças. Hoje, cerca de $20 \%$ da população da Região ainda depende de combustíveis sólidos poluidores para cozinhar e aquecer o ambiente. Aproximadamente 11 mil mortes causadas por doenças pulmonares obstrutivas crônicas (afetando mulheres de modo desproporcional) e 15 mil mortes de crianças abaixo de 5 anos, causadas por infecções agudas das vias aéreas inferiores, foram atribuídas ao fumo em ambientes fechados em 2000 (WHO, 2002). A violência, sob todas as formas, tornou-se um verdadeiro desafio para a saúde pública da Região. Cerca de 115.000 a 120.00o homicídios são registrados anualmente. A violência é a principal causa de morte em homens de 15 a 20 anos de idade, afetando significativamente os anos de vida prematuramente perdidos (Krug e col., 2002).

\section{Saúde, Meio Ambiente e as Metas de Desenvolvimento do Milênio}

As Metas de Desenvolvimento do Milênio (MDMs), cujos objetivos definidos são a redução da pobreza e a realização de intervenções para o desenvolvimento até 2015, reafirmam e atualizam os acordos celebrados em reuniões anteriores (Annan, 200o). Nesse contexto, questões relacionadas à saúde e ao meio ambiente tornaram-se novamente um dos objetivos centrais na luta contra a pobreza. A importância de garantir a segurança humana foi enfatizada através de metas específicas visando a integrar os princípios do desenvolvimento sustentável a políticas e programas dos países e a reverter a perda dos recursos ambientais. Estabeleceu-se o objetivo de reduzir pela metade a proporção de pessoas sem acesso sustentável a água potável segura e a saneamento básico até o ano de 2015, assim como o objetivo de melhorar significativamente as vidas de pelo menos 100 milhões de habitan- tes de favelas até o ano de 2020. Reduzir a dependência de combustíveis sólidos prejudiciais à saúde continua a ser um desafio importante.

Países na Região estão se esforçando para atingir as MDMs, promovendo novos tipos de parcerias entre governos, a sociedade civil, o setor privado e organizações internacionais, integrando gradualmente esses novos desafios a suas respectivas agendas políticas e planos de trabalho. Os países também estão cientes de que seus esforços até o presente foram insuficientes e de que é necessário acelerar o ritmo (Pan-American Health Organization, 2004). Há poucas dúvidas de que abordar os objetivos relacionados a água e saneamento, assim como reduzir a dependência de combustíveis nocivos, contribuirão para que se atinjam os objetivos cruciais referentes a mortalidade infantil.

\section{Um Esquema para a Ação}

À luz dos novos paradigmas e agendas que estão surgindo em saúde ambiental e desenvolvimento sustentável, novas abordagens e estratégias de trabalho adaptadas a necessidades presentes e futuras são necessárias. Estas incluem maior convergência entre programas e iniciativas desenvolvidas por agências do setor de saúde. Também são necessárias parcerias para reunir o capital tecnológico, humano, social, econômico e político que os países já possuem de maneira mais ordenada para reposicionar e fortalecer a saúde ambiental. 0 quadro de referência geral continua sendo o processo que integra desenvolvimento sustentável, segurança humana e direitos civis em suas várias dimensões políticas, ambientais, sociais e econômicas, expressas através de políticas públicas que satisfaçam adequadamente as necessidades distintas de diferentes grupos populacionais, melhorando, com isso, sua qualidade de vida, saúde e longevidade. (Fig. 1).

A redução e a prevenção de riscos têm sido uma das linhas de ação tradicionais em saúde ambiental. Essas iniciativas poderiam ser substancialmente fortalecidas adicionando-se outros programas à equação - programas para promover estilos de vida saudáveis e ambientes saudáveis. Essa sinergia entre controle de riscos e promoção de saúde (vista como fomentadora de fatores protetores) é o suporte que dá equilíbrio ao conteúdo dos novos programas de saúde ambiental. 
Fig I - Esquema de implementação para intervenções em saúde ambiental.

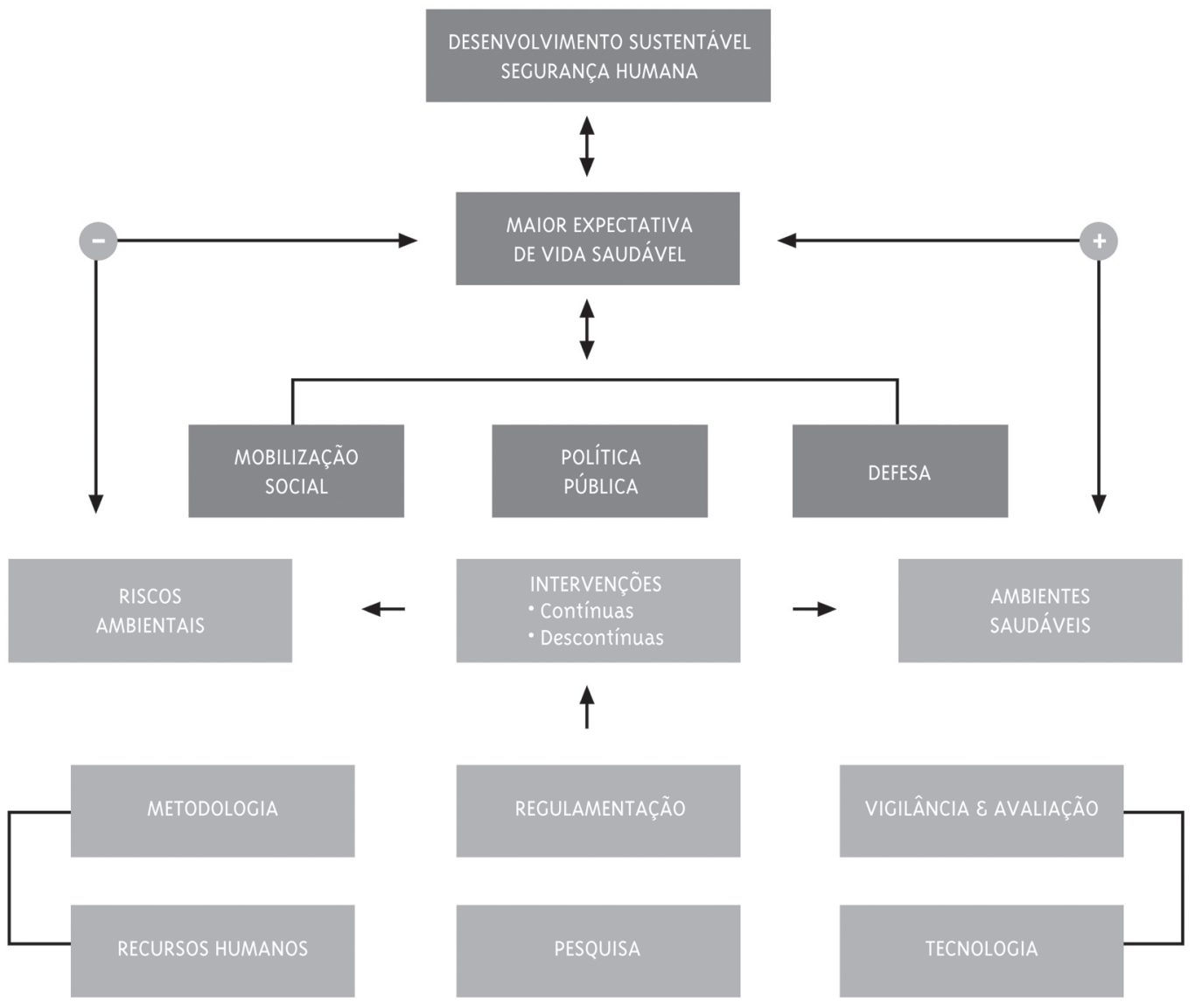

A saúde está no centro do desenvolvimento sustentável. Para que a saúde influencie positivamente o desenvolvimento, ela deve ser protegida contra riscos ambientais e promovida através de intervenções visando a construir ambientes saudáveis. Metodologia, tecnologia e pesquisa apropriadas, além de recursos humanos, vigilância e regulamentação, são as bases para intervenções adequadas.

Para garantir a eficiência e a eficácia de intervenções para controle de riscos e a promoção de espaços e comportamentos saudáveis, será necessário um conjunto abrangente de instrumentos. Será especialmente importante: a) aumentar o investimento em educação continuada para os responsáveis por instituições e programas, em todos os níveis; b) expandir as relações de trabalho entre a academia e os prestadores de serviços, para que metodologias integradas para a avaliação e o gerenciamento de riscos continuem sendo desenvolvidas e adaptadas; c) garantir reavaliações periódicas e realistas dos padrões e regulamentos que governam a saúde e o meio ambiente; d) alocar mais recursos para a melhoria contínua dos sistemas de informação voltados para identificação, análise, acompanhamento e controle oportunos de riscos sociais, ambientais e ocupacionais; e) promover o uso de tecnologia apropriada para aperfeiçoar a sensibilidade e a especificidade de programas de vigilância ambiental e da saúde; f) promover e encorajar projetos de pesquisa participativos; g) desenvolver programas de cooperação horizontal entre centros de referência, usando abordagens intersetoriais e interprogramáticas.

Em todos os níveis - regional, sub-regional e nacional - o setor saúde está reconhecendo cada vez mais a importância de se revisitar o conteúdo e as modalidades da cooperação técnica visando à saúde ambiental e ao desenvolvimento sustentável, promovendo uma sinergia entre as várias partes. Dessa maneira, contribuímos para aperfeiçoar resultados e atingir as metas e os objetivos acordados coletivamente. 


\section{Referências}

ANNAN, K. We the peoples: the role of the Nations United in the $21^{\text {st }}$ Century. New York: UN, 2000.

Disponível em: <http://www.un.org/millennium/sg/ report/>. Acesso em: 12 outubro 2006.

ANNAN, K. A more secure world: our shared responsibility. New York, 2004. Disponível em: $<$ http://www.un.org/secureworld >. Acesso em: 10 outubro 2006.

KRUG, E. G. et al (Ed.). World report on violence and health. Geneva: WHO, 2002.

LEFF, C. Ecology and capital: environmental rationality, participative democracy and sustainable development. Mexico, DF: $21^{\text {st }}$ Century, 1994.

ORGANIZATION OF AMERICAN STATES.

Hemispheric security mandates from the Third Summit of the Americas. Washington, DC, 2001. Disponível em: <http://www.summit-americas.org/ quebec-hem-security/hem-security-mainpageeng.htm>. Acesso em: 10 outubro 2006.
PAN-AMERICAN HEALTH ORGANIZATION.

Resolution CE134.R8: PAHO contribution to the fulfillment of the development goals of the United Nations Millennium Declaration. Washington, DC, 2004 .

UNITED NATIONS. World population prospects: the 2003 revision. New York, 2005. Disponível em: <http:/ lesa.un.org/unpp>. Acesso em: 20 janeiro 2007.

PAHO; WHO. Assessment of health effects of air pollution in Latin America and The Caribbean. Washington, DC, 2005.

WHO - WORLD HEALTH ORGANIZATION. World Health Report. Geneva, 2002.

WHO - WORLD HEALTH ORGANIZATION. Ecosystems and human well-being: health synthesis. Geneva, 2005.

WHO; UNICEF. For water life: making it happen. Geneva, 2005. 